\title{
A global petition: The potential significance of solidifying nutrition, lifestyle modification and epigenetic facets in our mundane practice
}

\author{
Nicholas Van Halm-Lutterodt ${ }^{1,2}$ and Yu Lun Xu ${ }^{1 *}$ \\ ${ }^{1}$ Department of Neurosurgery, Beijing Tiantan Hospital, Capital Medical University, China \\ ${ }^{2}$ Department Orthopedic Surgery and Neurosurgery, Keck Medical Center of USC, University of Southern California, USA
}

\section{Editorial}

Phylogenetically, we have been designed to properly function on biochemical fuel that is ultimately generated from complex biochemical processes beyond our control and of course exceeding the capability of the conscious mind. It could undoubtedly be appreciated that these biochemical fuel sources are greatly acquired through the food materials we ingest daily while our bodies execute eminent processes that cascade catabolic and anabolic reactions in an attempt to establish physiological assimilation and internal environment equilibrium under normally given conditions. It is also logical to understand that when one's musculoskeletal system gets worn-out or is deprived of the necessary functional needs (healthy nutrition, adequate physical activity and quality rest), global degenerative changes occur within the body to facilitate adaptation, a phased-period within which the body tries to incorporate the mind and the body for a global survival mode activation. That being said, it becomes clearly apparent how important healthy nutrition, adequate physical activity and rest are to the physiological function, most importantly the musculoskeletal system that forms the framework of the body. Within this nutritional domain, one of the most fascinating aspects that is less contemplated in our frequent approach to orthopaedics patients is the metabolic niche.

We encounter many orthopaedics patients both within the outpatient and inpatient settings who present with specialty-related problems but most often complicated with one or more co-morbidities that often vibrate from the nutritional-metabolic abyss. We have to observe this pitfall not as an unprofessional attributive factor but rather, an inclination to the nature of the demand and workloads that mount up over time for practitioners in the field and for that matter, a tilt towards specialty-specific related issues becomes more of a priority than intently addressing neighbouring issues like metabolic disarrays in orthopaedics patients unless otherwise the anticipated diagnoses are perceived to be osteoporosis or metabolic bone disease-focus.

We also can attest that, when there is malnutrition complicated with socio-economic factors such as stress and anorexic-bulimic components, underweight, overweight or obesity can result, and these abnormal weight outcomes complicated with metabolic syndromes exert great deal of health care burden and cost during delivery of care to orthopaedics patients as well.

More recently, there has been mounting evidence in the literature stemming from the cardio-metabolic discipline that discuss a subset of patient phenotype within the obese population. As we are all abreast with the fact that clinically, overweight and obesity are substantially associated with higher morbidity and mortality during patient care delivery, it is contradictory to conceive the notion that this phenotype set of patients, albeit obese, are dumbfoundingly metabolically healthy, hence the term; metabolically healthy obesity--(MHO) or metabolically normal obesity-- (MNO) have been coined to represent them.

The realization of this metabolically normal subset within the obese population is beginning to question our previous knowledge, awareness and understanding of the metabolic knowledge background and conceptualizations. It is certain that very little to none has been accomplished in the orthopaedics field regarding this interesting subject matter. The cause of this rabble-rousing finding within the obese population is yet to be explored in the orthopaedics field and hence requires collaborative and comprehensively integrated efforts that cut across every needing discipline.

If experts in the orthopaedics field are asked what the most dangerous item in a scenario of 'A plate mounted on a CHAIR with a delicious 3-level CHEESE BURGER' is? Assuming this question is thrown into a discussion forum for experts to share their comments and opinions, many would succumb to the notion that the dangerous item is the 3-level cheese burger. This is because we have so long been intellectually inclined and somewhat fixed to an orthodox philosophy that cheesy and oily foods are undoubtedly unhealthy. For instance, recent scientific reports of a studied population show that, in an aging population that tend to express lower serum low density lipoprotein cholesterol (LDL-c), they were rather at a health risk compared to those with higher serum levels of LDL-c. This is a clear example of the twist of knowledge and understanding of cholesterol and how essential it is for us to redress ourselves to meet exceptional expectations with evolving knowledge across every discipline that evaluate our clinical skills and delivery to all needing patients.

Averting this vulnerability in clinical practice requires reevaluation, re-education and updated knowledge base to align with the pathological findings of this fast pace of global economic development where everything appears to be so convenient and becoming almost

*Correspondence to: Yu Lun Xu, Department of Neurosurgery, Beijing Tiantan Hospital, Capital Medical University, China, Tel: +861067096507; E-mail: xuhenniu@qq.com

Received: December 12, 2018; Accepted: December 27, 2018; Published: December 31, 2018 
digital in both developed and developing countries. Actually, from a logical point of view, eating frequently isn't bad at all but how (quantity and quality) we eat and what we do after eating are that which predispose us to either a healthy or unhealthy metabolomic sequelae. In our opinion, the most dangerous item in the CHEESE BURGER scenario is the 'CHAIR'.

We often spend a lot of sedentary times in offices, homes, buses, trains, airplanes for longer periods and majority of peoples across the globe in the lower, middle and higher classes frequently invest less time engaging in physical activities like walking, bike riding, swimming, jogging and other aerobic exercises that healthily support cardiopulmonary function and necessary for precipitating a healthy metabolic outcomes that are essential for physio-biochemical processes in the body.

Research studies are strongly indicating the role of epigenetic susceptibility in such metabolic aberrations but interestingly, this facet falls under the biological niche that can be dynamically directed by lifestyle modifications through the emphasis of education and awareness programs, a demanded arena requiring vast integrated efforts from all medical, non-medical professionals as well as relevant organizations if we desire to halt this anticipated, accelerated extinction of the human race. The fact that there are some people in the general population albeit borderline or obese and yet demonstrate healthy or normal metabolic profiles as well as normal-weight population that are yet fraught with severe metabolic aberrations clearly indicate an interplay of adaptation, greatly attributed to pathophysiological epigenetic play role. This observation highlights the importance of the dynamic properties encoded within our genetic architecture, an epitome of resilience and potential warrant to educating our genes for individualized or well-tailored expression in a careful fashion to curb pathological abnormalities stemming from such epigenetic variations.

These efforts can be achieved in a condensed amount of time if we, the health professionals, non-governmental organizations as well as relevant government bodies re-evaluate our currently utilized interdisciplinary health care guidelines for necessary updates and amendments as it may be that, what we have previously perceived to be normal references during clinical evaluations may be requiring imminent adjustments to facilitate and enhance the quality of care delivered to all patients.

It would not be considered offensive at all to reverberate that it is about time that we medical professionals and experts in the orthopaedics field do also re-equip ourselves with this arm of interdisciplinary collaborative efforts to provide proper counselling and education by placing relayed emphases on nutrition and lifestyle modifications during patient-doctor consultations. Perhaps, some needless aggressive surgeries and treatments of inflamed joints, spinal disorders as well as related syndromes including Carpal Tunnel Syndrome (CTS) may be avoided when nutritional considerations and lifestyle changes are fiercely acknowledged in our day-to-day encounter with our patients.

Copyright: (C2018 Halm-Lutterodt NV. This is an open-access article distributed under the terms of the Creative Commons Attribution License, which permits unrestricted use, distribution, and reproduction in any medium, provided the original author and source are credited. 